\title{
Studies on the Polymerization of Acrolein Oxime. VI. Radiation-Induced Polymerization
}

\author{
Seizo Masuda, ${ }^{*}$ Harumi Tamal, ${ }^{*}$ Munetomo TorII, ${ }^{* *}$ \\ Masami TANAKA, ${ }^{* * *}$ and Tadatoshi OTA* \\ *Department of Applied Chemistry, Faculty of Engineering, \\ Tokushima University, Tokushima 770, Japan. \\ **Research and Development Division of Matsushita Denko Co., Ltd., \\ Yokkaichi 510, Japan. \\ ***Faculty of Pharmaceutical Sciences, Tokushima University of Arts \\ and Science, Tokushima 770, Japan.
}

(Received April 20, 1978)

\begin{abstract}
Radiation-induced polymerization and copolymerization of acrolein oxime are investigated in different solvents and at a wide range of temperature for obtaining information on the reaction mechanism. Acrolein oxime is polymerized ionically, irrespective of dryness of the sample. Arrhenius plots for the polymerization rate, which do not yield a linear relation, can be adequately approximated by two straight lines. An anionic mechanism is operative above the room temperature, while a cationic mechanism predominates below $-23^{\circ} \mathrm{C}$. The reaction in the intermediate temperature range proceeds by a competitive mechanism, and the rate of the anionic and cationic polymerizations becomes equal at the temperature near $-5^{\circ} \mathrm{C}$. The reaction rate is proportional to the square root of dose rate at room temperature and $-23^{\circ} \mathrm{C}$. On the basis of these data, it is proposed that the polymerization of acrolein oxime by $\gamma$-irradiation proceeds by free-ionic mechanisms.

KEY WORDS Acrolein Oxime / Radiation-Induced Polymerization / Ionic Mechanism / Activation Energies /
\end{abstract}

In the papers of this series, the followings were concluded: (1) Acrolein oxime (AOM) can not be polymerized radically, serving as an inhibitor for radical polymerizations of vinyl compounds. ${ }^{1}$ (2) AOM easily undergoes polymerization on heating, in which an anionic mechanism is operative. ${ }^{2,3}$ (3) AOM is also polymerized in the presence of a cationic or anionic catalyst. ${ }^{4,5}$ (4) Water has no influence on the rate of ionic polymerization of AOM.

It has been recognized in recent years that the polymerization of several vinyl compounds can occur by free-ionic mechanism. ${ }^{6}$ However, the extreme purification of monomers is required for experiment because the ionic polymerization is inhibited by trace amounts of impurity, especially water. AOM appears, from this point of view, to be a most interesting monomer, since the polymerization is neither initiated by any radical species nor inhibited by water. The present investigation is undertaken in order to elucidate the mechanism of the polymerization of AOM by $\gamma$-irradiation.

\section{EXPERIMENTAL}

AOM was prepared according to the method described previously ${ }^{7}$ and purified in three ways: It was, (a) distilled simply and pipetted, (b) distilled after drying over molecular sieves and pipetted, and (c) distilled in vacuo and admitted as such, into a glass ampoule. Acrylonitrile (AN) and solvents were purified by ordinary methods and distilled after drying over molecular sieves. The ampoule was cooled in liquid-nitrogen bath, degassed throughly and sealed in vacuo. After a definite time of irradiation, the contents were poured into a large excess of ether to precipitate the polymer. The rate of polymerization was evaluated by weighing the resulting polymer. The molecular weight of polymer was determined using dimethylfor- 
mamide (DMF) as the solvent at $70^{\circ} \mathrm{C}$ by a vapor pressure osmometer (Hitachi Perkin-Elmer, Model 115). The NMR spectrum was obtained with a JEOL PS-100 spectrometer $(100 \mathrm{MHz})$ at $21^{\circ} \mathrm{C}$ using deuterated dimethyl sulfoxide (DMSO- $d_{6}$ ) as the solvent and tetramethylsilane (TMS) as the internal standard. The polymer concentration was approximately $10 \%$ (wt/vol).

\section{RESULTS AND DISCUSSION}

The results of radiation-induced polymerization are summarized in Table I. The polymerization of $\mathrm{AOM}$, which is slower than that of AN or styrene (ST), is independent of additives such as $p$-benzoquinone (BQ), diphenylpicrylhydrazil (DPPH) and air. Homopolymerization of AN or ST, in which a free-radical mechanism is operative, is completely inhibited by the addition of $2-10-\mathrm{wt} \%$ AOM. However, the addition of over $20-\%$ AOM results in the formation of the polymer. Similar observations have been made in the thermal polymerization of AOM with vinyl compounds, in which the anionic mechanism is operative. ${ }^{2}$ Thus AOM can not be polymerized radically and

Table I. Radiation-induced polymerization of $\mathrm{AOM}, \mathrm{AN}$, and $\mathrm{ST}^{\mathrm{a}}$ at room temperature, at a dose rate of $2.75 \times 10^{5} \mathrm{r} / \mathrm{h}$

\begin{tabular}{|c|c|c|c|}
\hline $\begin{array}{c}{[\mathrm{AOM}]} \\
\mathrm{m} l\end{array}$ & $\begin{array}{c}\text { Comonomer, Additive } \\
\mathrm{m} l\end{array}$ & $\begin{array}{l}\text { Polymn } \\
\text { time, h }\end{array}$ & $\begin{array}{c}\text { Yield, } \\
\%\end{array}$ \\
\hline $5.0^{\mathrm{b}}$ & none & 4 & 2.5 \\
\hline 5.0 & & 4 & 2.4 \\
\hline 5.0 & $\mathrm{BQ}, 5 \times 10^{-3} \mathrm{~mol} /$ & $1 / l \quad 4$ & 2.2 \\
\hline 5.0 & DPPH, & 4 & 2.4 \\
\hline 5.0 & $5 \times 10^{-3} \mathrm{~mol} / l$ & 4 & 2.5 \\
\hline \multirow[t]{2}{*}{$5.0^{\mathrm{c}}$} & air & 24 & 1.5 \\
\hline & AN & & \\
\hline 0 & 5.0 & 4 & $>50$ \\
\hline 0.1 & 5.0 & 4 & 0 \\
\hline \multirow[t]{2}{*}{1.0} & 4.0 & 4 & 1.4 \\
\hline & ST & & \\
\hline 0 & 5.0 & 3 & $>50$ \\
\hline 0.1 & 5.0 & 3 & 0 \\
\hline 0.5 & 5.0 & 3 & 0 \\
\hline 1.0 & 4.0 & 3 & 0.1 \\
\hline
\end{tabular}

a $\mathrm{AOM}$ and comonomers were purified by means of (a).

${ }^{b}$ Purified by means of (b).

c Polymerization was carried out at $0^{\circ} \mathrm{C}$. may serve as an inhibitor for radical polymerization of vinyl compounds by $\gamma$-irradiation. The dryness of the sample does not affect the yield of polymer, and hence subsequent experiments were carried out after purification by (a).

The polymerization of AOM at a dose rate of $1.84 \times 10^{5} \mathrm{r} / \mathrm{h}$ was carried out at a temperature

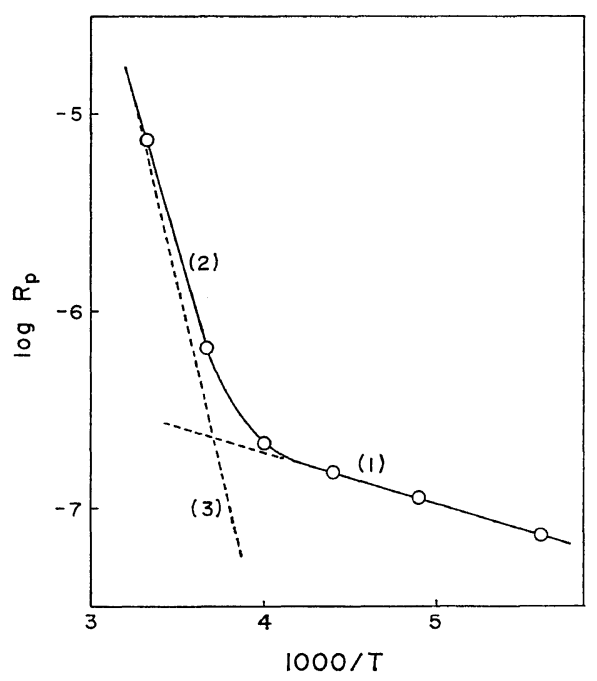

Figure 1. Arrhenius plot for the polymerization of AOM in MTHF solution: $[\mathrm{AOM}]=6.86 \mathrm{~mol} / l$; dose rate, $1.84 \times 10^{5} \mathrm{r} / \mathrm{h}$.

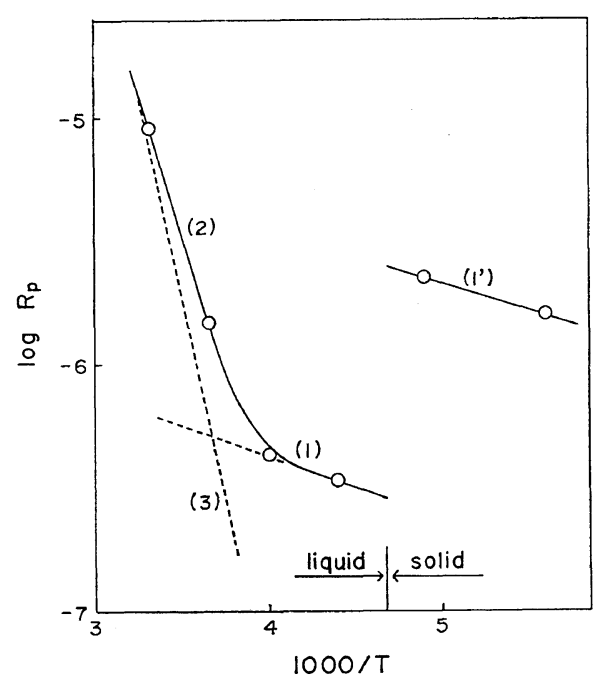

Figure 2. Arrhenius plot for the polymerization of $\mathrm{AOM}$ in $\mathrm{BuCl}$ solution: $[\mathrm{AOM}]=6.86 \mathrm{~mol} / l$; dose rate, $1.84 \times 10^{5} \mathrm{r} / \mathrm{h}$. 


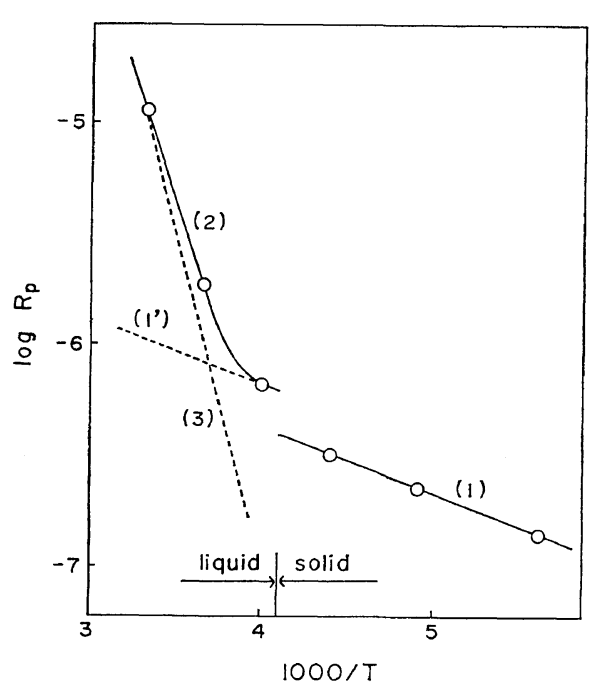

Figure 3. Arrhenius plot for the bulk polymerization of AOM: $[\mathrm{AOM}]=13.74 \mathrm{~mol} / l$; dose rate, $1.84 \times$ $10^{5} \mathrm{r} / \mathrm{h}$.

ranging from room temperature to $-95^{\circ} \mathrm{C}$, and tert-butyl chloride $(\mathrm{BuCl})$ and methyl tetrahydrofuran (MTHF) were used as the solvents. Temperature dependence of the rate of polymerization is shown in Figures 1-3. In all systems, Arrhenius plots were found not to be linear. Though the curves appear to have a linear portion above $0^{\circ} \mathrm{C}$, they are not straight lines in the strict sense because the polymerization proceeds via two mechanisms to be described later. In addition, the curves discontinued at the freezing point except for the case of MTHF solution; the MTHF solution is in the liquid state over a whole temperature range, while liquid AOM and the DCE solution of AOM are transformed into the solid state at $-45^{\circ}$ and $-70^{\circ} \mathrm{C}$, respectively.
For the AOM polymerization in MTHF solution, the Arrhenius plot was linear at the temperature below $-23^{\circ} \mathrm{C}$, the activation energies calculated being $1.2 \mathrm{kcal} / \mathrm{mol}$, which was approximately equal to that for the hydrochloric acidcatalyzed polymerization. ${ }^{5}$ On the basis of this fact and the copolymerization data which will be described below, it may be seen that the polymerization proceeds by the cationic mechanism at a temperature below $-23^{\circ} \mathrm{C}$. On the other hand, there is a rapid increase in the rate above this temperature. A logarithmic plot of the difference between the overall rate and the rate of the cationic polymerization gave a dotted line (3). From the slope of the line (3), the activation energy was evaluated to be $17.5 \mathrm{kcal} / \mathrm{mol}$, which was approximately equal to that for the butyllithium (BuLi)catalyzed polymerization. ${ }^{4}$ Therefore, the dotted line (3) corresponds to the Arrhenius plot for the anionic polymerization; that is, the anionic polymerization is predominant at increased temperature.

In the AOM polymerization in DCE solution, an Arrhenius curve similar to the case of the MTHF solution was obtained except for a discontinuity at the freezing point. The solid-state polymerization was faster than the liquid-state polymerization ranging from $-23^{\circ}$ to $-45^{\circ} \mathrm{C}$, but the similar value of activation energies was evaluated. The solid-state polymerization is also thought to proceed via the cationic mechanism. The activation energies for the cationic and anionic polymerization were calculated from the slope of the straight lines (1) and (3), respectively, and the values obtained are summarized in Table II.

A plot of $\log R_{\mathrm{p}} v s .1 / T$ for the bulk polymerization, as with the polymerization in DCE solu-

Table II. Activation energies for the AOM polymerization by $\gamma$-irradiation

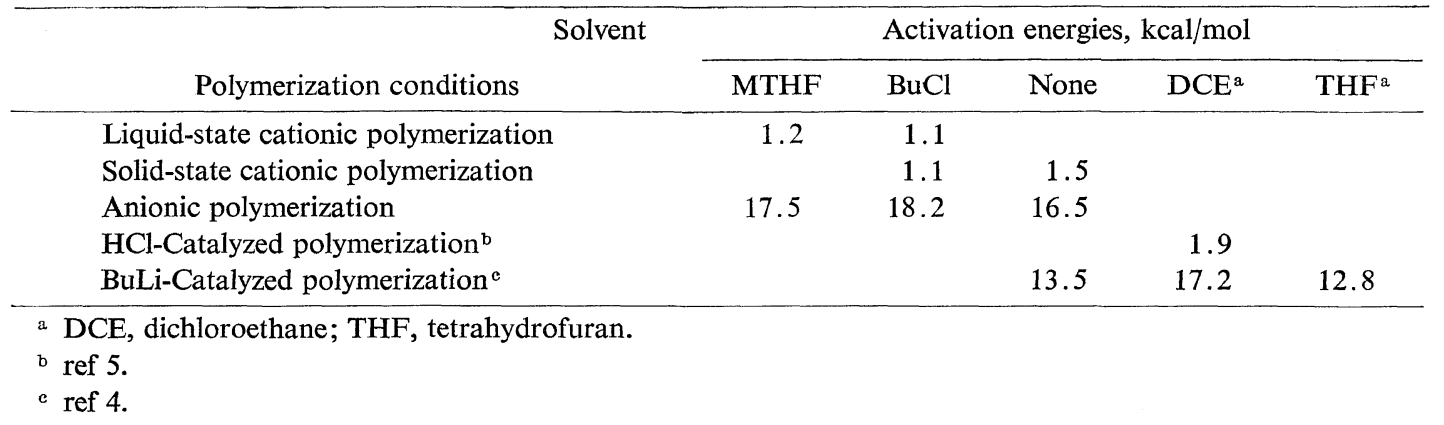


Table III. $G(\mathrm{M})$ values for the AOM polymerization at a dose rate of $1.84 \times 10^{5} \mathrm{r} / \mathrm{h}$

\begin{tabular}{ccrrrrrr}
\hline & & \multicolumn{7}{c}{$G$ Value } \\
\cline { 3 - 8 } Solvent & $G(\mathrm{M})$ & \multicolumn{1}{c}{$\mathrm{rt}^{\mathrm{a}}$} & $0^{\circ} \mathrm{C}$ & $-23^{\circ} \mathrm{C}$ & $-45^{\circ} \mathrm{C}$ & $-70^{\circ} \mathrm{C}$ & $-95^{\circ} \mathrm{C}$ \\
\hline \multirow{3}{*}{ MTHF } & $G_{\mathrm{T}}(\mathrm{M})^{\mathrm{b}}$ & 140.2 & 12.7 & 4.2 & 2.9 & 2.1 & 1.4 \\
& $G_{\mathrm{C}}(\mathrm{M})^{\mathrm{c}}$ & 5.8 & 4.8 & 3.8 & 2.9 & 2.1 & 1.4 \\
& $G_{\mathrm{A}}(\mathrm{M})^{\mathrm{d}}$ & 134.4 & 7.9 & 0.4 & & & \\
$\mathrm{BuCl}$ & $G_{\mathrm{T}}(\mathrm{M})$ & 172.8 & 28.7 & 8.3 & 6.6 & 42.5 & 30.8 \\
& $G_{\mathrm{C}}(\mathrm{M})$ & 11.7 & 9.7 & 7.5 & 6.6 & 42.5 & 30.8 \\
& $G_{\mathrm{A}}(\mathrm{M})$ & 161.1 & 19.0 & 0.8 & & & \\
\hline \multirow{3}{*}{ None } & $G_{\mathrm{T}}(\mathrm{M})$ & 223.3 & 33.9 & 12.8 & 9.7 & 4.2 & 2.7 \\
& $G_{\mathrm{C}}(\mathrm{M})$ & 30.9 & 16.1 & 11.6 & 9.7 & 4.2 & 2.7 \\
& $G_{\mathrm{A}}(\mathrm{M})$ & 192.4 & 17.8 & 1.2 & & & \\
\hline
\end{tabular}

a rt, room temperature.

b Total $G(\mathrm{M})$ value.

c $G(\mathrm{M})$ value for cationic polymerization.

d $G(\mathrm{M})$ value for anionic polymerization.

tion, discontinued at the freezing point, but the solid-state cationic polymerization was slower than the liquid-state cationic polymerization. The apparent activation energy for the solid-state cationic polymerization was calculated from the slope of straight line (1), and that for the liquidstate cationic polymerization was assumed to be equal to that for the solid-state one.

Contrary to the expectation that the cationic mechanism is operative in a $\mathrm{BuCl}$ solution, while the anionic mechanism is predominant in MTHF solution, the temperature is found to be the only factor governing the reaction; the anionic mechanism is operative above room temperature, while the cationic mechanism is below $-23^{\circ} \mathrm{C}$. The reaction in the intermediate temperature range proceeds by a competitive mechanism, and the rate of the anionic and cationic polymerizations becomes equal at a temperature near $-5^{\circ} \mathrm{C}$. The $G$ values of reacted AOM $(G(\mathrm{M}))$ are calculated as given in Table III.

Figure 4 shows the logarithmic plots of polymerization rate and dose rate. Based on these results, the empirical equation for the rate of polymerization $\left(R_{\mathrm{p}}\right)$ was derived as a function of dose rate (I).

$$
\begin{array}{ll}
R_{\mathrm{p}} \propto I^{0.54} & \text { at room temperature } \\
R_{\mathrm{p}} \propto I^{0.51} & \text { at }-23^{\circ} \mathrm{C}
\end{array}
$$

The establishment of half-power dependence suggests that radiation-induced polymerization of AOM proceeds by a free-ionic mechanism. Ueno,

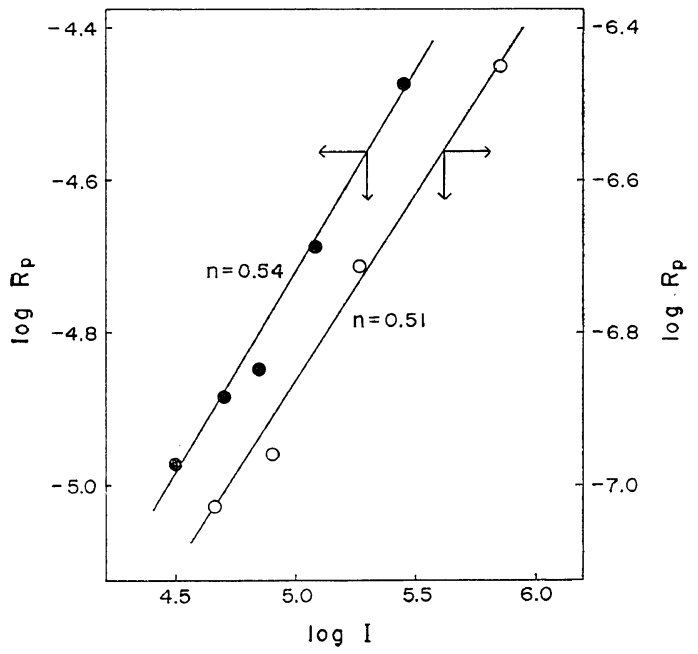

Figure 4. Dose-rate dependence of the rate of bulk polymerization at room temperature $(0)$ and at $-23^{\circ} \mathrm{C}$ (O): $[\mathrm{AOM}]=13.74 \mathrm{~mol} / \mathrm{l}$.

et al., ${ }^{8}$ have published that reaction order with respect to dose rate approaches one half as the polymerization system becomes free from impurity, especially water. As described previously, ${ }^{5}$ since AOM can not be polymerized radically and since water has no influence on the rate of ionic polymerization of AOM, the half-power dependence of the polymerization rate on the dose rate seems to be due to bimolecular termination of free cations and free anions.

Figure 5 illustrates the radiation-induced copoly- 


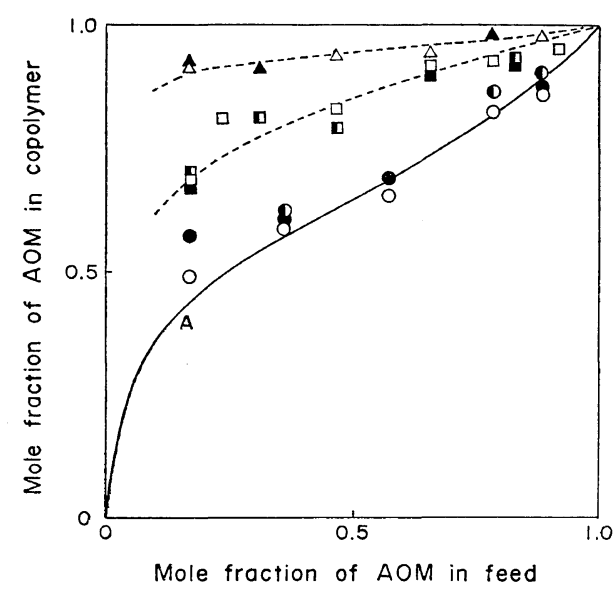

Figure 5. Copolymerization diagrams for AOMAN system: dose rate, $1.84 \times 10^{5} \mathrm{r} / \mathrm{h}$; yields were up to $5 \%$; circule, at room temperature; square, at $0^{\circ} \mathrm{C}$; triangle, at $-23^{\circ} \mathrm{C}$; solvent, $\operatorname{MTHF}(\bullet, \mathbf{0}, \mathbf{\Delta}), \mathrm{BuCl}$ $(\boldsymbol{O}, \mathbf{\square})$ and none $(O, \square, \triangle)$; solid line $A$, monomercopolymer composition curve for $\mathrm{BuLi}$, catalyzedcopolymerization at $0^{\circ} \mathrm{C} .^{2}$

merization of AOM with AN, where copolymer compositions depend not only on the kind of solvent used but also on the reaction temperature. Comparison of the composition diagrams for radiation-induced copolymerization at room temperature and BuLi-catalyzed copolymerization at $30^{\circ} \mathrm{C}$ (solid line) gave excellent agreement, but the content of AN in copolymer decreases with a decrease in temperature because AN does not polymerize cationically. The conclusion drawn from copolymerization data is in agreement with that described above, in which temperature alone governs the reaction, and the anionic polymerization is predominant above room temperature, while the cationic polymerization is operative below $-23^{\circ} \mathrm{C}$.

Although a detailed discussion of the polymerization scheme can not be given at present, the following cationic and anionic species are presumed to be the active species.

Formation of ion radical:

$$
\begin{aligned}
& \mathrm{AOM} \longrightarrow(\mathrm{AOM}) . \oplus+\mathrm{e} \ominus \\
& \mathrm{AOM}+\mathrm{e} \ominus \longrightarrow(\mathrm{AOM}) . \ominus
\end{aligned}
$$

Ion radical-molecule reaction:

Since the iminoxy-type radical of AOM is stable, ${ }^{1}$ the reaction of ion radical and AOM molecule is as follows.

$$
\begin{gathered}
(\mathrm{AOM}) . \oplus+\mathrm{AOM} \rightarrow\left(\mathrm{CH}_{3}-\stackrel{\oplus}{\mathrm{C}} \mathrm{H} \leftrightarrow \mathrm{CH}_{3}-\mathrm{CH}=\mathrm{CH}-\stackrel{\oplus}{\mathrm{C}}\right) \\
+\mathrm{CH}_{2}=\mathrm{N}-\mathrm{OH}-\mathrm{CH}=\mathrm{N}-\mathrm{O} \cdot \\
(\mathrm{AOM}) . \ominus+\mathrm{AOM}-\left(\mathrm{CH}_{3}-\stackrel{\mathrm{C}}{!} \mathrm{H} \leftrightarrow \mathrm{CH}_{3}-\mathrm{CH}=\mathrm{CH}-\stackrel{\ominus}{\mathrm{N}}\right) \\
\stackrel{\mathrm{CH}=\mathrm{N}-\mathrm{OH}}{\mathrm{O}} \\
+\mathrm{CH}_{2}=\mathrm{CH}-\mathrm{CH}=\mathrm{N}-\mathrm{O} .
\end{gathered}
$$

The polymers obtained by the polymerizations at $-70^{\circ} \mathrm{C}$ and room temperature are soluble in DMF and water, and in DMF, respectively. The molecular weight of the former is about 400 and that of the latter is about 1000. In the cationic polymerization by $\gamma$-irradiation as well as in the catalyzed cationic polymerization, a 1,2-addition predominates to yield a water-soluble polymer which contains considerable pendant oxime groups. The NMR spectrum of the polymer has a peak assigned to methyl protons, suggesting that the chain-transfer reaction involves the elimination of proton from the growing chain end. On the other hand, the polymer obtained at the room temperature has a peak assigned to $\mathrm{CH}_{2}=\mathrm{CH}-$ $\mathrm{CH}=\mathrm{N}-\mathrm{O}-$ in the NMR spectrum. It suggests that the anionic polymerization by $\gamma$-irradiation is practically initiated by the AOM anion $\left(\mathrm{CH}_{2}=\right.$ $\mathrm{CH}-\mathrm{CH}=\mathrm{N}-\mathrm{O}^{\ominus}$ ) produced by a chain transfer to a monomer.

Acknowledgment. A part of this work was done under Visiting Researchers Program of Kyoto University Research Reactor Institute. The authors are grateful to Assistant Professor Hitoshi Yamaoka for helpful suggestions.

\section{REFERENCES}

1. S. Masuda and T. Ota, Kobunshi Ronbunshu, 31, 61 (1974).

2. S. Masuda and T. Ota, Polym. J., 9, 459 (1977).

3. S. Masuda and T. Ota, Polym. J., 9, 465 (1977).

4. S. Masuda, M. Tanaka, and T. Ota, Polym. J., 10, 387 (1978).

5. S. Masuda and T. Ota, Polym. J., 10, 397 (1978).

6. For example, R. B. Taylor, and F. Williams, J. Am. Chem. Soc., 91, 3728 (1969); R. C. Potter and D. J. Metz, J. Polym. Sci., Part A-1, 9, 441 (1971); 
S. Masuda, H. TAmai, M. ToriI, M. TAnaka, and T. Ota

H. Yamaoka, H. Mori, K. Hayashi, and S. Okamura, J. Polym. Sci., Part B, 7, 371 (1969); Ka. Hayashi, Y. Yamazawa, T. Takagaki, F. Williams, K. Hayashi, and S. Okamura, Trans. Faraday Soc., 63, 1489 (1967).
7. S. Masuda, H. Kihara, and T. Ota, Nippon Kagaku Kaishi, 608 (1974).

8. K. Ueno, F. Williams, K. Hayashi, and S. Okamura, Trans. Faraday Soc., 63, 1478 (1967). 